\title{
Convulsions in pregnancy-not always eclampsia role of neuro-imaging in diagnostic dilemma
}

\author{
M Vijayasree ${ }^{1}$, GV Murali ${ }^{2}$ \\ Department of Obstetrics ${ }^{1}$, Department of Neuro Sciences ${ }^{2}$ \\ Mamata General Hospital, Khammam, Andhrapradesh
}

\begin{abstract}
Background: Convulsions in pregnancy is always considered and treated as Eclampsia unless otherwise proved. Eclampsia is associated with increased risk of maternal death varying from $1.8 \%$ to $14 \%$ in developed countries. Despite availability of Intensive care units and improved antenatal care, some women still die from Eclampsia. Cerebral complications are the major cause of death in eclampsia patients. Hypercoagulopathy of pregnancy is a high risk factor for these patients in respect to development of cerebro vascular thrombosis and ischaemic strokes. Eclampsia patients who are refractory to the routine treatment have been found to have various Central Nervous System pathological conditions amenable to the medical treatment. Objective: To study the neuro pathophysiology behind a seizure in pregnancy and to reduce the morbidity associated with it. To study the role of neuro-imaging in patients with convulsions in pregnancy not responding to treatment with magnesium sulphate $\left(\mathrm{MgSo}_{4}\right)$. Methods: It was a prospective study design which included 50 antenatal mothers. All patients were admitted in the eclampsia room with history of convulsions and all of them were put on $\mathrm{MgSo}_{4}$ therapy and Antihyperertensives. The patients who were refractory to the treatment such as having recurrent convulsions despite therapy with $\mathrm{MgSo}_{4}$ were selected for neuro- imaging with Computed Tomography scan. Neuro-imaging was done using Phillips Tomoscan CT scanner where slices of $10-\mathrm{mm}$ thickness were taken through the entire brain in the trans-axial plane. Abdomen shielding was done with lead shield to prevent radiation hazard in the antenatal period.The results were documented and analysed using appropriate statistical method. Results: The CT scanning report revealed: Cerebral oedema (30/50), Encephalopathy (10/50), Intra Cranial Haemorrhage (02/50) Cerebral infarction (01/50), Cortical venous sinus thrombosis (01/50), Tuberculomas (01/50), Neurocysticercosis (01/50) and Hydrocephalus (01/50). Three patients

\footnotetext{
Address for correspondence

Dr. M. Vijayasree

Telangana Hospitals, Balaji Nagar, Khammam Andhra Pradesh, India

E-mail: hospitalstelangana@gmail.com
}

out of 50 had normal CT scan report. Conclusion: Patients with convulsions in pregnancy who were refractory to the treatment with $\mathrm{MgSo}_{4}$ and
\end{abstract}


Antihypertensives have been found to have very significant and morbid CNS pathological conditions. Neuro imaging in these patients have done a pivotal role in identifying the abnormality and rectifying it with medical means which had definitely improved patients conditions and have reduced morbidity and mortality.

Keywords: Convulsions, neuro-imaging, pregnancy

\section{Introduction}

Eclampsia is defined as occurrence of one or more convulsions in pregnant woman with hypertension and proteinuria that cannot be attributed to any other cause. In developing countries like India, eclampsia leads to complications in about 1-100 out of 1700 deliveries. Despite availability of intensive care units and improved antenatal care, some women still die from eclampsia. Cerebral complications are the major cause of death in eclampsia patients, but the neuro pathophysiology of eclamptic seizure still remain undiscovered. ${ }^{1}$

Eclampsia itself along with hypercoagulopathy of pregnancy is a high risk factor for development of Cerebral venous sinus thrombosis and intracranial haemorrhage ${ }^{2-6}$. Although eclampsia affects variety of organs, cerebrovascular involvement is the major cause of death in eclampsia patients ${ }^{4,6}$, there has been considerable debate as to whether the neurological symptoms of eclampsia arise from the over auto regulation that causes vasospasm and ischaemia or from hyper perfusion that causes cerebral oedema formation ${ }^{7}$.

To describe neurological involvement in eclampsia, two theories have been proposed $^{6}$. Theory of vasospasm: In severe hypertension and in eclampsia, cerebral auto regulation comes into play which causes cerebral vasoconstriction. This vasospasm is believed to cause local anoxic damage to endothelium of capillaries and disruption of blood brain barrier, which leads to cerebral oedema. Theory of dilatation: Sudden fluctuation in blood pressure exerts greater pressure on capillary walls and leads to extra vasation of proteins and fluids causing pericapillary ring haemorrhages. Most common finding on CT scan in eclampsia patients is generalized cerebral oedema and features suggestive of hypertensive encephalopathy ${ }^{8}$, neuro imaging can be very helpful in eclampsia patients who do not respond to conventional treatment with $\mathrm{MgSO}_{4}$ and Antihypertensives ${ }^{4}$.

CT scan findings in eclampsia patient have been found mainly transient cortical and sub cortical white matter hypo densities which 
could be due to hypoxia or oedema ${ }^{9}$. These lesions correspond to mainly water shed areas of circulation where anterior, middle and posterior cerebral arteries meet, it is in this area where the earliest breakthrough in auto regulation occurs ${ }^{4,7,10}$. Convulsions in pregnancy should be always treated as eclampsia unless otherwise proved. But in cases where the patients who are not responding to the standard $\mathrm{MgSo}_{4}$ regimens or deteriorating in spite of treatment we have to evaluate the patient for other intra cranial causes of convulsions. Neuro imaging plays a vital role in such diagnostic dilemmas. So we have undertaken this study to evaluate the role of neuro imaging in patients with convulsions in pregnancy who are refractory to conventional treatment with $\mathrm{MgSo}_{4}$.

\section{Methods}

This was a prospective study conducted at the department of obstetrics and gynaecology and department of neuro sciences in Mamata General Hospital, Khammam, Andhra Pradesh, India. A total of 50 patients with convulsions in pregnancy who were refractory to conventional treatment for eclampsia with $\mathrm{MgSO}_{4}$ were included in this study. Patients were selected by simple random sampling. On admission -patient's detailed history was elicited. History of epilepsy in the past was enquired. General, systemic and obstetric examination was done. Appropriate investigations were ordered including pregnancy induced hypertention profile and coagulation profile and all the patients were treated with conventional $\mathrm{MgSo}_{4}$ regimen. Those patients who did not respond to Inj$\mathrm{MgSO}_{4}$ (blood level within therapeutic range of 4-7 meq/1) were labelled as refractory eclampsia ${ }^{4}$. In all these patients neuro imaging was done using computed tomography.

\section{Results}

Table 1: Distribution of patients by age $(n=50)$

\begin{tabular}{|l|l|l|}
\hline \multicolumn{1}{|c|}{ Age of patient (years) } & \multicolumn{1}{|c|}{ No. of patients } & \multicolumn{1}{c|}{ Percent } \\
\hline $15-20$ & 23 & $46 \%$ \\
\hline $21-25$ & 15 & $30 \%$ \\
\hline $26-30$ & 07 & $14 \%$ \\
\hline $31-35$ & 05 & $10 \%$ \\
\hline Total & 50 & $100 \%$ \\
\hline
\end{tabular}


Distribution of patients by age $(n=50$.): In our study, $46 \%$ of the patients with convulsions were in the teenage group. It supports the genetic theory of development of pre eclampsia where alloimmunity between maternal and fetal tissues is the predisposing factor $^{4}$. Only $10 \%$ of them were above 30 years of age.

Table 2: Distribution of patients by type of eclampsia $(n=50)$

\begin{tabular}{|l|l|l|}
\hline Type of eclampsia & No. of patients & $\%$ \\
\hline Ante- partum & 38 & $76 \%$ \\
\hline Post- partum & 12 & $24 \%$ \\
\hline Intra- partum & Nil & Nil \\
\hline Total & 50 & $100 \%$ \\
\hline
\end{tabular}

Distribution of patients by type of eclampsia $(\mathrm{n}=50)$ : Although globally postpartum eclampsia is more common ${ }^{2}$, our study came across $76 \%$ Anteparum Eclampsia patients. Developing countries like India still have inadequate. Antenatal care and unsupervised home deliveries ${ }^{11}$. In a recent survey almost $50-60 \%$ deliveries still occur at home and are conducted by untrained dais.

Table 3: Distribution of patients in relation to neurological symptoms $(n=50)$

\begin{tabular}{|l|l|l|}
\hline $\begin{array}{l}\text { Neurological } \\
\text { symptoms }\end{array}$ & No. of patients & $\%$ \\
\hline Convulsions & 30 & $60 \%$ \\
\hline
\end{tabular}

\begin{tabular}{|l|l|l|}
\hline Deep coma & 10 & $20 \%$ \\
\hline Altered sensorium & 07 & $14 \%$ \\
\hline Cortical blindness & 03 & $06 \%$ \\
\hline Total & 50 & $100 \%$ \\
\hline
\end{tabular}

Distribution of patients in relation to neurological symptoms ( $n=50)$ : Majority $60 \%$ of the patients presented with convulsions either focal or generalized. $20 \%$ of them went into deep coma; in these patients the prognosis was poor. $14 \%$ of them had altered sensorium and $6 \%$ had cortical blindness.

Table 4: Distribution of patients in relation to type of $\mathrm{mgso}_{4}$ regimen used $(n=50)$

\begin{tabular}{|l|l|l|}
\hline $\mathrm{MgSo}_{4}$ regimen & No. of patients & Percent \\
\hline Low Dose & 40 & $80 \%$ \\
\hline Pritchard & 10 & $20 \%$ \\
\hline Total & 50 & $100 \%$ \\
\hline
\end{tabular}

Distribution of patients in relation to type of $\mathrm{MgSO}_{4}$ regimen used $(\mathrm{n}=50)$ : Low dose regimen has been devised by Dr. Sardesai et al, also called as Solapur Regimen. In this regimen, initially 4 grams Intra Venous Bolus Injection of $\mathrm{MgSO}_{4}$ dissolved in $20 \mathrm{cc}$ of normal saline is given slowly over 20 minutes followed by 2 grams Intra Muscularly every 3 hours in alternate buttocks. Principle behind giving 2 grams as maintenance dose is that Indian women have less body mass index as compared to her western counter part and so Pritchard regimen is not suited to Indian women where overdose toxicity can be a 
possibility. In this study $80 \%$ of the patients were treated with low dose $\mathrm{MgSO}_{4}$ where as $20 \%$ of them needed Pritchard regimen since they were obese.

Table 5: Distribution of patients on glasgow coma scale on admission $(n=50)$

\begin{tabular}{|l|l|l|}
\hline Glasgow coma scale & No of patients & $\%$ \\
\hline$<10$ & 10 & 20 \\
\hline $11-12$ & 10 & 20 \\
\hline $13-14$ & 30 & 60 \\
\hline$>14$ & Nil & Nil \\
\hline Total & 50 & 100 \\
\hline
\end{tabular}

categorized the patients on the basis of glasgow coma scale where we found it to be extremely useful for individualization of the treatment. With the help of the same, we were able to register the recovery of patients also. There were ten patients in our study in which the scale was <10: In all these patients, we have found central nervous system pathological conditions such as severe cerebral edema, intra cerebral haemorrhage etc., majority of the patients were in between 13-14 Glasgow coma score $(60 \%)$. None of them were more than 14 glasgow coma score.

Distribution of patients on glasgow coma scale on admission $(n=50)$ : We have

Table 6: Distribution of patients in relation to CT scan findings in patients with convulsions $(\mathrm{n}=50)$

\begin{tabular}{|l|l|l|l|}
\hline S.N. & \multicolumn{1}{|c|}{ Finding } & No. of patients & \multicolumn{1}{c|}{ Percentage } \\
\hline 1 & Cerebral oedema & 30 & $60 \%$ \\
\hline 2 & Encephalopathy & 10 & $20 \%$ \\
\hline 3 & Intra cerebral haemorrhage & 02 & $04 \%$ \\
\hline 4 & Cerebral infarction & 01 & $02 \%$ \\
\hline 5 & Cortical venous sinus thrombosis & 01 & $02 \%$ \\
\hline 6 & Tuberculomas & 01 & $02 \%$ \\
\hline 7 & Neuro-cysticercosis & 01 & $02 \%$ \\
\hline 8 & Hydrocephalous & 01 & $02 \%$ \\
\hline 9 & Normal & 03 & $06 \%$ \\
\hline & Total & 50 & $100 \%$ \\
\hline
\end{tabular}

Distribution of patients in relation to CT scan findings in patients with convulsions $(n=50)$ :
We have found neuro-imaging playing a pivotal role in all patients with convulsions 
who were not responding to the conventional management with $\mathrm{MgSO}_{4}$ and antihypertensives. Cerebral edema was found in $60 \%$ of the patients and encephalopathy syndrome in $20 \%$ of them. Intra cerebral haemorrhage was found in $4 \%$ of the cases. Other conditions like cerebral infarction, cortical venous sinus thrombosis, tuberculoma, neuro-cysticercosis and hydrocephalous was found in two percent of the patients respectively on CT scan. This prompted us to proceed with the treatment into the right direction. We have also found a very interesting case of neurocysticercosis which has duly responded to albendazole.

Maternal outcome: All the patients recovered well after institution of appropriate treatment. However, patient with cerebral venous sinus thrombosis had protracted recovery and required mechanical ventilation in the intensive care unit.

Fetal outcome: Induction of labour was done in all the patients with convulsions during pregnancy after initial stabilisation. With prostaglandins most of the neonates born were healthy $(35 / 50)$, but some of them required neonatal intensive care unit support $(07 / 50)$ and mechanical ventilation (03/50). Five out of fifty babies were stillborn due to extreme prematurity and other complication such as abruptio placenta.

\section{Discussion}

In our study we have come across some interesting cases which we have diagnosed early and treated them effectively in our hospital with the help of CT scan and MRI studies. I would like to discuss four interesting case reports.

\section{Case reports}

Case 01:

A 26 years old primigravida with 32 weeks of pregnancy came with history of four episodes of convulsions. On admission her Blood Pressure was $150 / 90 \mathrm{~mm} \mathrm{Hg}$. Proteinuria was present. She was started on injection $\mathrm{MgSO}_{4}$ (Pritchard regimen).In spite of the treatment repeated episodes of convulsions were present. CT scan was done on the patient who showed multiple calcified tuberculomas in the entire brain. She was treated with Anti Tuberculous Treatment and the patient responded well.

Case 02:

A Primigravida aged 22 years was admitted with history of one generalized tonic clonic convulsion at home. On admission, her Blood Pressure was $180 / 110 \mathrm{~mm} \mathrm{Hg}$ with $3+$ proteinuria. Patient was put on $\mathrm{MgSO}_{4}$ and Antihypertensives. Patient was induced with Prostaglandin $E_{2}$ Gel. After few hours, she suddenly became drowsy and was unresponsive to verbal commands. CT scan 
was done on the patient and was found to have large left occipital intra cerebral haemorrhage. Patient shifted to medical Intensive care unit and was put on mannitol, Anti Epileptics and mechanical ventilation. Gradually she was weaned off from ventilator after five days. She was discharged from the hospital after three weeks in good general condition.

\section{Case 03:}

A 29 years old third gravida with full term pregnancy was referred from a local private hospital with history of focal convulsions. On admission her Blood pressure was 140/100 $\mathrm{mm}$ of $\mathrm{Hg}$ with minimal proteinuria. Patient was conscious and well oriented on admission .she experienced few episodes of convulsions in spite of $\mathrm{MgSO}_{4}$ regimen. CT scan was done and she was found to have neurocysticercotic lesions throughout the brain she was treated with mannitol, albendazole and anti epileptics and she was free of convulsions within a week and was discharged after fifteen days on anti epileptics.

\section{Case 04:}

A 19-year-old Primiparous woman presented with history of convulsions in the postpartum period. She was complaining of blindness and severe headache. Her Blood pressure was $160 / 110 \mathrm{~mm} \mathrm{Hg}$.she was put on
Antithypertensives and $\mathrm{MgSo}_{4}$ regimen. We have done Magnetic Resonance Imaging on her and she was found to have Encephalopathy. Patient was put on mannitol and steroids. Her condition improved within 15 days.

\section{Conclusion}

A convulsion in pregnancy is a major cause of maternal mortality in India along with Heamorrhage and Infection. $\mathrm{MgSO}_{4}$ treatment in patients with convulsions have shown to reduce morbidity and mortality as been proved by many studies. In our study, neuro imaging in patients with convulsions during pregnancy who were refractory to the conventional treatment with $\mathrm{MgSO}_{4}$ were found to have various central nervous system abnormalities such as venous infarct, cortical venous sinus thrombosis and infections such as tuberculomas/neurocysticercosis etc. Neuro imaging in these patients have done a pivotal role in identifying the abnormality and rectifying it with medical means which has surely improved patient's condition and have reduced the maternal mortality. So my study concludes that patients with convulsions in pregnancy unresponsive to conventional treatment should be screened by neuro imaging (computed tomography scan /magnetic resonance imaging brain) to exclude serious morbid central nervous system pathology. 


\section{References}

1. Villar MA, Sibai BM. Eclampsia. In: Arias F, editor. Obstetrics and gynaecological clinics of North America. Philadelphia: High risk Pregnancy. 1988; 356-77.

2. Leitch CR, Cameron AD, Walker JJ. The changing pattern of eclampsia over a 60 - year period. $\mathrm{Br} J$ Obstet Gynaecol . 1997; 104:917-22 (Retrospective Series;1259 patients ).

3. Mass JL, Lamy C. Stroke in pregnancy in the postpartum period. In: Ginsberg MD,Bogousslavsky J, editors. Cerebrovascular disease: pathophysiology, diagnosis and Management. Malden: Blackwell Sciences. 1988; 1684-97.

4 Kanki T, Mihara F, Nakanoh. Diffusion weighted images and vasogenic edema in Pregnancy. Obstet Gynaecol. 1999; 93:821-3.

5. Zeeman G, Flankestein JJ, Twickler DM. Cerebral infarction in eclampsia. Am J Obstet Gynaecol. 2002; 100:140.
6. Manfredi M, Baltramello A. Eclamptic encephalopathy, neuro- imaging and Pathological Consideration. Acta Neurol Scand. 1997; 96:277-82.

7. Swartz RB, Feske SK, Polak JF, et al. Pre-eclampsia, eclampsia; clinical and neuro radiological correlates and insights in the pathogenesis of hypertensive encephalopathy. Radiology. 2000; 217:317-76.

8. Zhu XW. Cerebral lesions in severe PIH : 61 cases study with computed tomographic scan. Zonghua Chan Ke Za Chi.1993;28(5)275-7,313.

9. Lauska DJ. Peripartum Stroke and intracranial vascular thrombosis in National Hospital Admissions. Obstet Gynacol 1997; 89:413-8.

10. Millez J, Dahoun A, Boudraa M, et al. Computed tomography brain in Eclampsia. Am J Obstet Gynacol. 1990; 75(6):975-80.

11. Naidu K, Modly J. SPECT, CT scan, and TCD findings in eclampsia. $\mathrm{Br} J$ Obstet Gynaecol. 1997; 104(10):116272. 